Mater. Sci. Bull., Vot. 1. No. 1, May 1974. pp. 4y-57, (2) Printed in India.

\title{
Permeability spectrum of garnets
}

\author{
OM PRAKASH, R AIYER and C M SRIVASTAVA \\ Department of Physics. Indian Institute of Technology, Bombay 400076
}

MS received 20 November 1978

\begin{abstract}
A detailed study of permeability spectra of polycrystalline pure and Aland Gd-substituted garnets has been carricd out for different values of grain diameter and magnetisation. The spectra are of three types-resonance, relaxed and mixed. It has been shown that the resonance spectra occur when the closure dcmains are absent and the domain wall is anchored at the grain boundary. The relaxed spectrum is found for domains having closure fux while the mixed spectrum is an indication of simultaneous presence of grains with and without closure domains.
\end{abstract}

\section{Introduction}

The permeatility spectra of fertites and gareets has been extensively studied in the past (Rado et al 1950; Brown and Gravel 1955: Smolenski et al 1961; Verweel 1962; Malinofsky and Babbit 1961: Globus 1962, 1975). However a number of experimental results have not been satisfactorily explained. This has been partly due to difficulties in the preparation of samples having parameters like magnetisation and microstructure varying over a wide range to enable a consistent phenomenological model to emerge. In this work we attempl to study the behaviour of the permeability spectrum using samples of pure and substituted yttrium iron garnets with saturation magnetisation varying from 90 to $18 \mathrm{co}$ gauss. Further, grain size has been varied from $1 \mu \mathrm{m}$ to $15 \mu \mathrm{m}$ using hot pressing and conventional sintering techniques. The results of these investigations generally agree with current theory (Globus 1975) that permeability linearly increases with grain size. Our studies show that the conclusions of Globus $(1975,1976)$ regarding dependence of the nature of the permeability spectrum on grain size are not true when $4 \pi M$ and microstructure vary over a wide range. According to him the shape of the permeability spectrum is relaxed for uniform and homogeneous grain size samples irrespective of the size of the grain and is of the resonance type when the grain size is inhomogeneous. Our studies indicate that the grain size is the predominant factor which controls the nature of the permeability spectrum. For constant magnetisation we have observed resonance spectra for small grain size semples $\left(D \leqslant D_{01}\right)$ and a relaxed spectrum for large grain size samples $\left(D \geqslant D_{02}\right)$. We also observe a distinct transition region $\left(D_{01} \leqslant D \leqslant D_{02}\right)$ in which the shape is of the mixed type for the intermediate grain size samples. This transition region 
is found to depend on the magnetisation of the samples and the critical grain diameters $D_{112}$ and $D_{022}$ are a linear function of magnetisation, for large number of domain walls.

An explanation for the observed behaviour of the permeability spectrum is given on the basis of the number of domain walls within a grain of the polycrystalline sample. It is found that the resonance spectrum appears when closure domains are absent due to the smallness of the grain size while the relaxed spectra occur when for larger grains closure domains are present.

\section{Experimental}

Sample preparation: Pure $\mathrm{Y} / \mathrm{G}\left(\mathrm{Y}_{3} \mathrm{Fe}_{5} \mathrm{O}_{12}\right)$ and $\mathrm{Al}$ - and $\mathrm{Gd}$-substituted iron garnets $\left(\mathrm{Y}_{3-2} \mathrm{GA}_{1} \mathrm{Fe}_{i-2} \mathrm{Al}, \mathrm{O}_{12} ; x=0, y=0 \cdot 2,0 \cdot 4,0 \cdot 65,0 \cdot 8 ; y=0, x=0.5\right.$. $1,1 \cdot 4,2.3)$ were prepared in disk form. High purity $(>99.9 \%)$ chemicals in nearly stoichiometric proportions were coprecipitated and further processed using standard ceramic techniques. High density pellets of $9-10 \mathrm{~mm}$ diameter and 3 to $4 \mathrm{~mm}$ thickness were prepared by hot pressing the pre-sintered powder of submicron size. The sintered samples were ultrasonically cut into toroidal form, polished, thermally etched and re-annealed in oxygen atmosphere for investigations like microstructure and permeability studies.

The increase in grain size was obtained by subsequent firing and soaking at elevated temperatures using the normal sintering method.

Metallographic studies Were carried out to determine the grain size of single phase samples. The method used for permeability studies is described elsewhere (Srivastava et al 1976).

\section{Analysis and discussions}

\subsection{Low frequency permeability}

The low frequency permeability is observed (Globus 1975) to increase linearly with grain diameter. The contribution to permeability from domain wall translation is given by

$$
\mu^{\prime}-1=\frac{3 \pi}{4} M_{m} \ldots \frac{D}{\sigma_{w}\left(1-\frac{w^{2}}{w_{0}^{2}}\right)},
$$

where $M_{\delta}$ is the saturation magnetisation, $\sigma_{w}$ is the wall energy per unit area, $w$ is the operating frequency, $w_{0}$ is the domain wall resonance frequency and $D$ is the grain diameter. This equation can be derived from the equation of motion for the domain wall and agrees with the results obtained by Globus $(1975,1976)$ for the susceptibility at low frequencies.

In figure 1, we give the plot of $\left(\mu^{\prime}-1\right) / D$ against $M_{s}^{2}$ for the normal-sintered $\mathrm{Gd}_{z} \mathrm{Y}_{3-x} \mathrm{Fe}_{5} \mathrm{O}_{12}$ samples. The measurements are made at $10 \mathrm{kHz}$ which are small compared to the resonance frequency $f_{0}(\sim 1 \mathrm{MHz})$. In figure 2 , we give a similar plot for the hot-pressed Y-Gd-garnets. We have chosen this composition so that $\sigma_{t o}$ is fairly constant when $4 \pi M_{\text {s }}$ is varied from 90 to 1800 . Neglecting $\left(w / w_{Q}\right)^{2}$ 


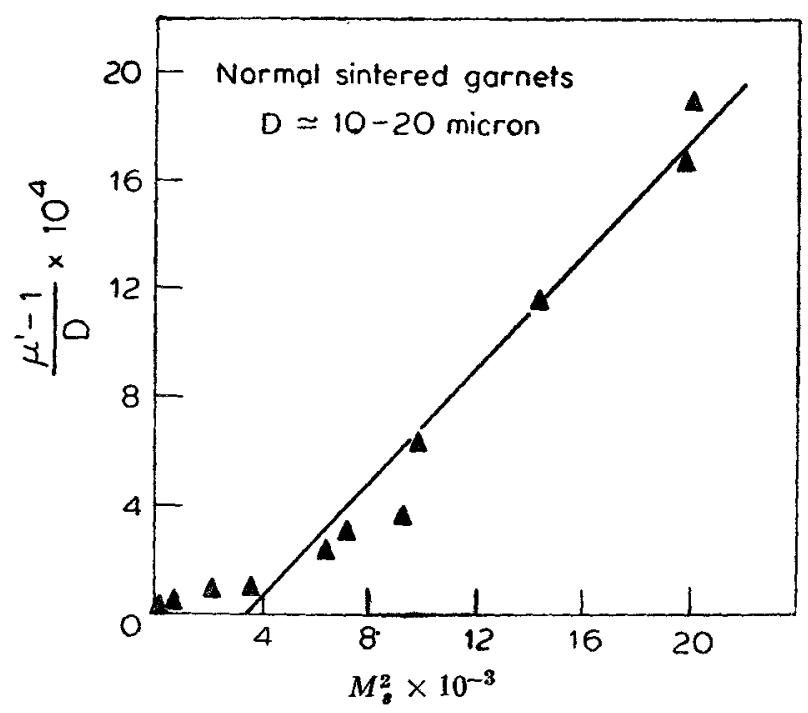

Figure 1. $\left(\mu^{\prime}-1\right) / D$ versus $M_{8}^{2}$ plot for normal sintered Gd-substituted garnets with large grain size, $D>10 \mu \mathrm{m}$.

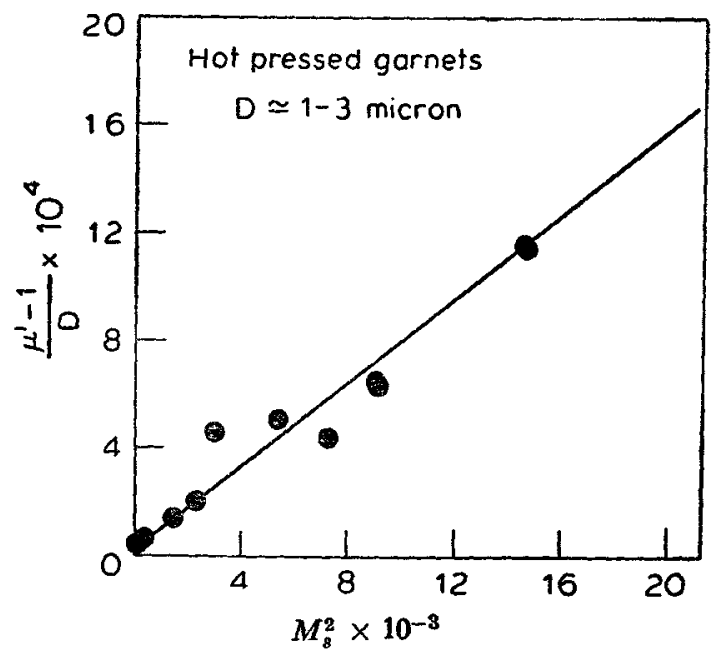

Figure 2. $\left(\mu^{\prime}-1\right) / D$ versus $M_{s}^{2}$ plot for hot-pressed Gd-substituted garnets with small grain size, $D<3 \mu \mathrm{m}$.

compared to 1 we obtain from the plot the value of $\sigma_{w}$ which is equal to 0.23 $\mathrm{erg} / \mathrm{cm}^{2}$ for the normal sintered and $0.30 \mathrm{erg} / \mathrm{cm}^{2}$ for the hot-pressed samples. An estimate of $\sigma_{10}$ can be obtained from the relation (Chickazumi and Charap 1964)

$$
\sigma_{\varphi}=2\left[\left(J_{A D} S^{2} \pi^{2} K\right) / a\right]^{1 / 2}
$$


where $J_{A D}$ is the exchange constant for the $A-D$ interaction in the garnet system (assumed to be the dcminant interaction), $K$ is the anisotropy constant and $a$ is the distance between $A$ and $D$ site spins. Taking $J_{A D}=30.4^{\circ} \mathrm{K}, K=6.2 \times 10^{3}$ $\mathrm{erg} / \mathrm{cm}^{2}, a=3.46 \AA$ and $S=5 / 2$ appropriate for the pure YIG, we obtain $\sigma_{\omega}=0.27 \mathrm{erg} / \mathrm{cm}^{2}$. The experimental values of $\sigma_{\omega}$ thus agree with theory.

\subsection{Permeability spectrum}

We have studied the permeability spectrum from $10 \mathrm{kHz}$ to $1000 \mathrm{MHz}$ for different values of grain diameter for several compositions of YGd and YAI garnets. We observe that for the same composition a resonance spectrum is obtained for small grain diameters and a relaxed spectrum for large grain diameters. Our observation thus contradicts the results of Globus $(1975,1976)$ that relaxed spectrum is observed for samples with uniform grain diameter and resonance behaviour when grain size is inhomogeneous. In figure 3 we give the permeability spectrum of the normal sintered YIG having a grain diameter of $12 \mu \mathrm{m}$. This spectrum is of a relaxed type. In figure 4 is given the plot of the spectrum of pure YIG for grain diameter of $1.9 \mu \mathrm{m}$ which is of the resonance type. The spectrum of Al-YIG for grain diameter $6.5 \mu \mathrm{m}$ is given in figure 5. This is of mixed type. Extensive permeability spectra measurements have been made on several compositions of different grain diameters and it is found that all the spectra can be classified in one of the above thiee categories. Our results are summarised in table 1 . In figure 6 we give the plot of the nature of the permeability spectrum as a function of magnetisation and the number of domain walls within a grain of radius $R$.

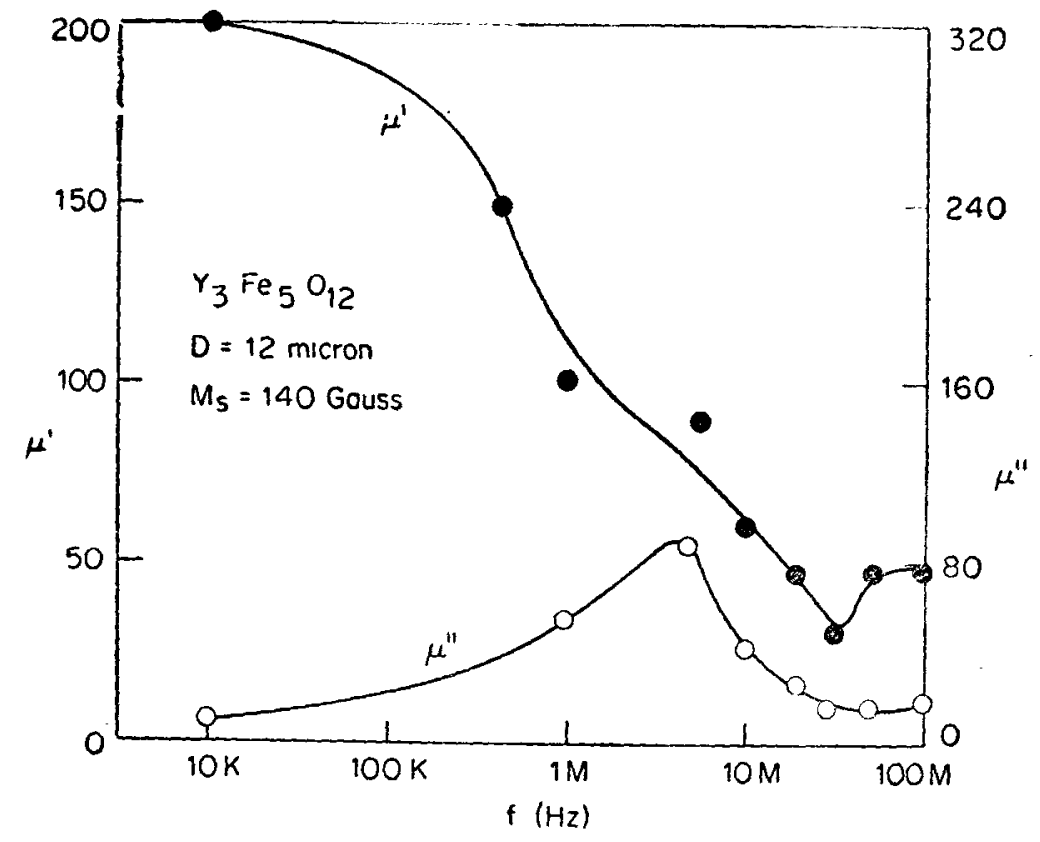

Figure 3. Permeability spectra of pure YTG for grain size $D=12$ micron showing relaxed type behaviour. 


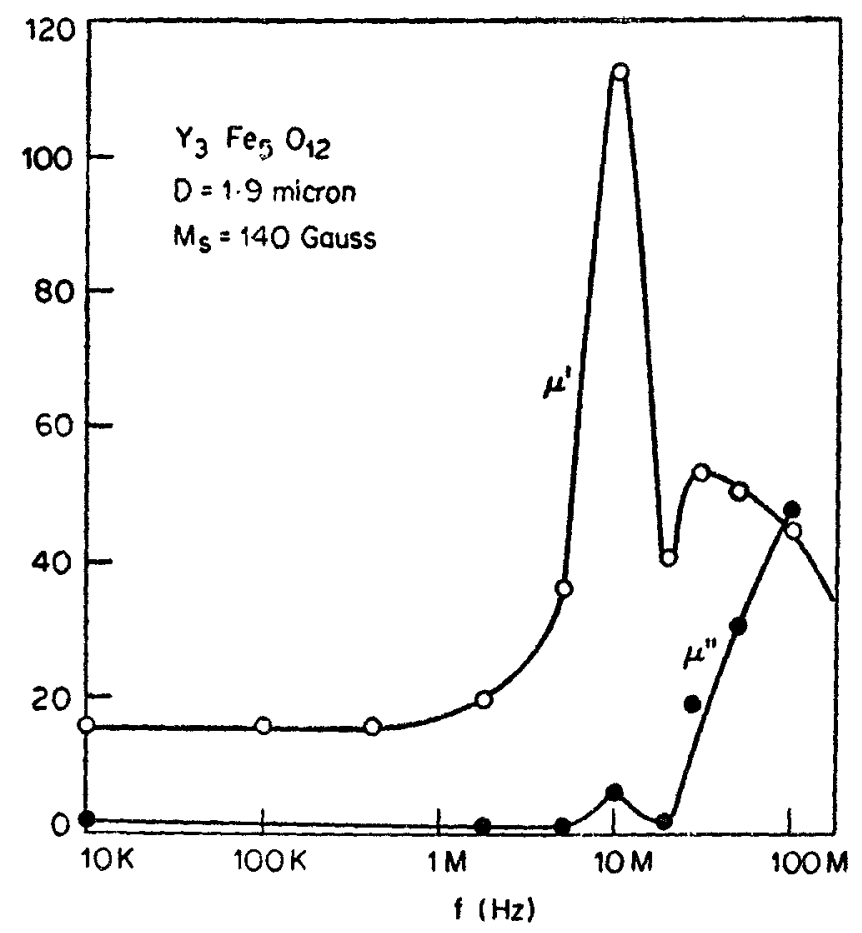

Figure 4. Permeability spectra of pure YIG for grain size $D=1.9$ micron showing resonance type behaviour.

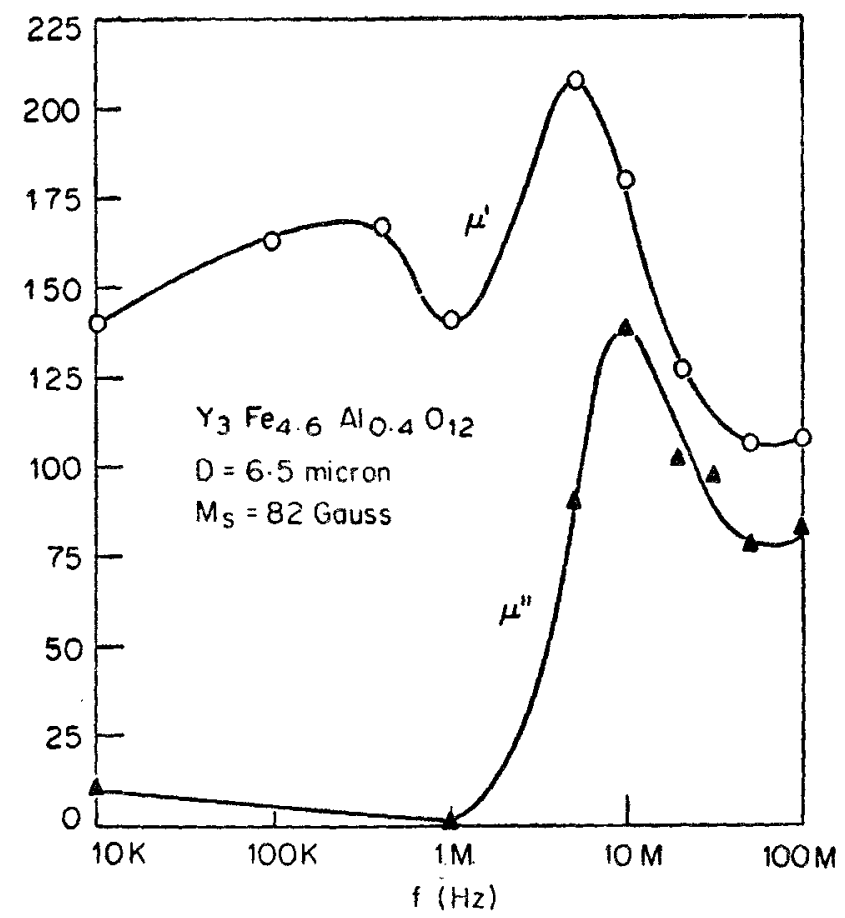

Figure 5. Permeability spectra of Al-substituted garnet for grain size $D=6.5$ micron showing mixed type behaviour. 
Table 1. Nature of the permeability spectrum of varying grain size samples of pure YIG and Gd- and Al-substituted iron garnets. The $M_{s}$ and $\mu^{\prime}$ at $10 \mathrm{kHz}$ are also given.

\begin{tabular}{|c|c|c|c|c|}
\hline Composition & $\begin{array}{c}M \\
\text { Gauss }\end{array}$ & $\begin{array}{l}\text { Grain size } \\
\text { micron }\end{array}$ & $\begin{array}{c}\mu^{\prime} \text { at } \\
10 \mathrm{kHz}\end{array}$ & $\begin{array}{l}\text { Nature of permea- } \\
\text { bility spectrum }\end{array}$ \\
\hline $\mathrm{Y}_{3} \mathrm{Fe}_{5} \mathrm{O}_{12}$ & 140 & 1.9 & 32 & Resonance \\
\hline $\mathrm{Y}_{3} \mathrm{Fe}_{\overline{5}} \mathrm{O}_{12}$ & 139 & $3 \cdot 0$ & 60 & Resonance \\
\hline $\mathrm{Y}_{3} \mathrm{Fe}_{\overline{9}} \mathrm{O}_{18}$ & 141 & $8 \cdot 5$ & 155 & Relaxed \\
\hline $\mathrm{Y}_{3} \mathrm{Fe}_{5} \mathrm{O}_{12}$ & 140 & 12 & 200 & Relaxed \\
\hline$Y_{2 \cdot 6} \mathrm{Gd}_{0.4} \mathrm{Fe}_{5} \mathrm{O}_{12}$ & 121 & $2 \cdot 5$ & 30 & Resonance \\
\hline $\mathrm{Y}_{2} \mathrm{G}_{\mathrm{d}_{1}} \mathrm{Fe}_{5} \mathrm{O}_{12}$ & 94 & 2 & 13 & Resonance \\
\hline $\mathrm{Y}_{2} \mathrm{Gd}_{1} \mathrm{Fe}_{5} \mathrm{O}_{\mathbf{1} 2}$ & $95 \cdot 5$ & 3 & 20 & Resonance \\
\hline $\mathrm{Y}_{2} \mathrm{GdFe}_{5} \mathrm{O}_{12}$ & $95 \cdot 5$ & 6 & 40 & Resonance \\
\hline $\mathrm{Y}_{2} \mathrm{GdFe}_{5} \mathrm{O}_{12}$ & 97 & 13 & 56 & Mixed \\
\hline $\mathrm{Y}_{2} \mathrm{GdFe}_{5} \mathrm{O}_{12}$ & $105 \cdot 5$ & 20 & 74 & Relaxed \\
\hline $\mathrm{Y}_{1.7} \mathrm{Gd}_{1.3} \mathrm{Fe}_{5} \mathrm{O}_{12}$ & 56 & 2 & 10 & Resonance \\
\hline$Y_{1 \cdot 7} \mathrm{Gd}_{1 \cdot 3} \mathrm{Fe}_{5} \mathrm{O}_{12}$ & $70 \cdot 6$ & $6 \cdot 5$ & 30 & Resonance \\
\hline$Y_{1 \cdot 7} \mathrm{Gd}_{1 \cdot 2} \mathrm{Fe}_{5} \mathrm{O}_{1}$ & 66 & $14 \cdot 8$ & 35 & Relaxed \\
\hline $\mathrm{Y}_{1 \cdot 3} \mathrm{Gd}_{1 \cdot 7} \mathrm{Fe}_{5} \mathrm{O}_{22}$ & 44 & $12 \cdot 6$ & 12 & Mixed \\
\hline $\mathrm{Y}_{1.6} \mathrm{Gd}_{1.4} \mathrm{Fe}_{5} \mathrm{O}_{12}$ & 73 & $1 \cdot 8$ & 10 & Resonance \\
\hline$Y \mathrm{Gd}_{2} \mathrm{Fe}_{5} \mathrm{O}_{12}$ & 48 & $2 \cdot 8$ & $6 \cdot 5$ & Resonance \\
\hline $\mathrm{YGd} \mathrm{Fe}_{5} \mathrm{O}_{12}$ & $48 \cdot 3$ & 15 & $13 \cdot 5$ & Mixed \\
\hline $\mathrm{Y}_{8} \mathrm{Fe}_{4 \cdot 8} \mathrm{Al}_{0 \cdot 2} \mathrm{O}_{19}$ & 113 & $1 \cdot 4$ & 32 & Resonance \\
\hline $\mathrm{Y}_{3} \mathrm{Fe}_{4 \cdot 8} \mathrm{Al}_{0.2} \mathrm{O}_{12}$ & 120 & $5 \cdot 5$ & 106 & Mixed \\
\hline $\mathrm{Y}_{2} \mathrm{Fe}_{1 \cdot 0.4 \mathrm{Al}_{0.4} \mathrm{O}_{12}}$ & 82 & $1 \cdot 7$ & 24 & Resonance \\
\hline $\mathrm{Y}_{8} \mathrm{Fe}_{4.8} \mathrm{Al}_{0.4} \mathrm{O}_{18}$ & 82 & $7 \cdot 5$ & 90 & Relaxed \\
\hline $\mathrm{Y}_{2} \mathrm{Fe}_{4 \cdot 35} \mathrm{Al}_{0 \cdot 85} \mathrm{O}_{12}$ & 60 & $1 \cdot 7$ & 18 & Resonance \\
\hline $\mathrm{Y}_{3} \mathrm{Fe}_{4.8} \mathrm{Al}_{0.8} \mathrm{O}_{12}$ & $13 \cdot 8$ & $4 \cdot 6$ & 19 & Mixed \\
\hline
\end{tabular}

It is observed that the boundaries for resonance-to-mixed and mixed-to-relaxed spectra are well defined. An explanation for these results in terms of the equilibrium number of domain walls within a grain boundary with and without closure domains is given below. The resonance spectra are shown to arise when closure domains are absent and the wall is pinned to grain boundary. The relaxed spectra occur when closure domains are present.

The dynamics of domain walls under external time varying fields has been discussed by many authors (Globus 1975; Rado 1953; Smit and Wijn 1959; Srivastava et al 1976). The equation of motion for domain walls is

$$
m_{w} \dot{z}+\beta_{n} \dot{z}+a_{w} z=2 M_{i} H,
$$

where $m_{w}$ is the effective mass per unit area of the domain wall, $\beta_{w}$ is the damping constant and $\alpha_{\infty}$ is the stiffness constant. As discussed by Srivastava et al (1976) 


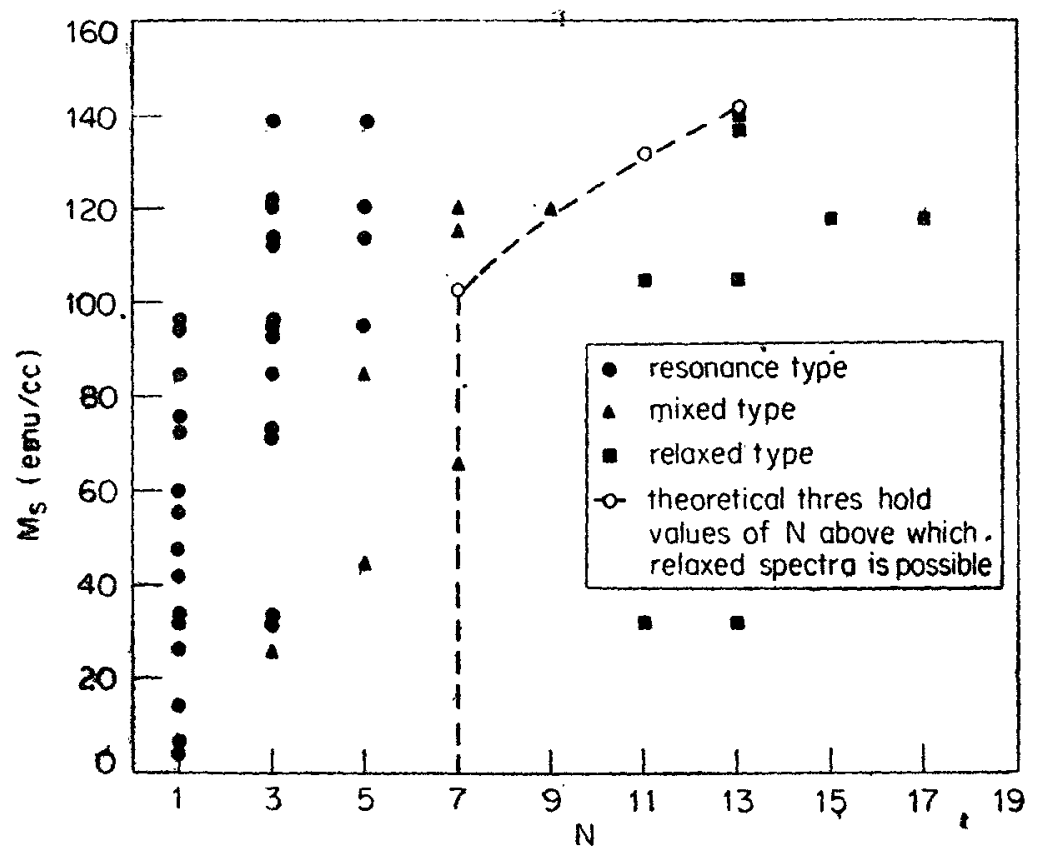

Figure 6. Nature of permeability spectra as a function of magnetisation $\left(M_{s}\right)$ and number of domain walls (N) within a grain of polycrystalline garnet sample. Three types of permeability spectra have been observed.

the analysis of the resonance spectrum can be carried out using (3) in terms of the resonance frequency $w_{r}$ and the relaxation frequency $\Omega$ which are given by

$$
w_{r}=\left(w_{0}^{2}-\Omega^{2}\right)^{1 / 2}=\left(\frac{a_{w 0}}{m_{10}}-\frac{\beta_{w 0}^{2}}{4 m_{w 0}^{2}}\right)^{1 / 2}
$$

and $\Omega=\beta_{10} / 2 m_{\omega}$. The data obtained using this analysis will be published later.

The analysis of the relaxed spectrum can be carried out using (3) if the term in $m_{w}$ or the term in $a_{w}$ is negligible. As discussed by Kittel and Galt (1956), the inertia rerm is negligible in ferromagnetic materials. So far no explanation has been offered for the smallness of the inertia term when the relaxed spectrum is observed.

It is observed from figure 6 that relaxed spectrum is obtained for increasing values of $n$ with increase in $M_{s}$. To understand this result we have to calculate the equilibrium number of domain walls which can exist within a grain of radius $R$. This can be obtained by minimising the total energy consisting of the wall energy and the magnetostatic energy. If there are $(2 n+1)$ walls within a sphere of radius $R$, the total energy is given by

$$
\begin{aligned}
w= & \pi R^{2}\left[(2 n+1)-\frac{n(2 n+1)}{3(n+1)}\right] \sigma_{*} \\
& +\frac{1}{2(n+1)} \frac{1}{2}\left(\frac{4}{3} \pi M_{*}\right)\left(\frac{4}{3} \pi R^{3} M_{*}\right)
\end{aligned}
$$

Minimising $w$ with respect to $n$, the total number of domain walls, $N$ within a grain is given by 


$$
N=(2 n+1)=2\left[\frac{\pi}{3} \frac{M_{0}^{2} R}{\sigma_{w}}-\frac{1}{4}\right]^{1 / 2}-1
$$

The condition for the formation of one domain wall is given by $N=1$ which leads to a critical grain radius $R$, given by

$$
R_{\mathrm{e}}=\frac{15}{4 \pi} \frac{\sigma_{\mathrm{w}}}{M_{\mathrm{s}}}
$$

Equation (6) indicates that the equilibrium number $N$ of domain walls reduces almost linearly with $M$, for a constant grain radius $R$ provided $\sigma_{w}$ is constant. For small values of $M_{*}, N$ would tend to one which would mean that there will be only one domain wall. As the value of $M_{4}$ is increased the number of domain walls within the grain increases. In case a grain is single domain or has just one domain wall, closure domains cannot be formed. As the number of walls increases the possibility of the formation of closure domains increases.

For the relaxed spectrum we expect the inertial term to be insignificant. This can occur when the closure domains are present so that the domain wall can move without any inertia and with a constant velocity as soon as it is subjected to an external force. In such cases the low frequency permeability will be large. On the other hand, if the domain wall is anchored at the grain boundary, as will be the case without closure domains, the inertial term will be significant, leading to a resonance type spectrum and small low frequency permeability. From this we conclude that the relaxed spectrum is obtained when the closure domains are present and resonance spectrum when there are no closure domains.

The condition for the formation of closuie domains is obtained by minimising the total energy consisting of the wall energy and the anisotropy energy. The anisotropy energy is calculated using the following assumptions:

(i) As we move from the centre, the thickness of the closure domain varies as

$$
d=k(1-r / n) l
$$

where $r$ is the $r$-th domain counted from the centre, $l$ is the domain width and $k$ is a constant. This form takes into account the fact that at the two extremes there are no closure domairs within a spherical grain.

(ii) Within a disk type domain assumed herein, the volume of the closure domain is approximately

$$
2 \pi\left(R^{2}-r^{2} l^{2}\right)^{112} l . d \text {. }
$$

(iii) Anisotropy energy varies as a function of magnetisation according to the relation

$$
K_{\mathrm{a}}=K_{0}\left(M_{\mathrm{a}} / M_{0}\right)
$$

where $K_{0}$ and $M_{0}$ refer to the anisotropy energy and magnetisation of YIG and $K_{\text {a }}$ and $M$, refer to that of the substituted garnets.

(iv) The number of domains is large, $n \geqslant 1$. Using these assumptions we have

$$
w_{\mathrm{anls}}=4 \pi \frac{R^{3} k}{n(n+1)^{3}} \frac{K_{0} M_{s}}{M_{0}} \sum_{r=1}^{n}\left[(n+1)^{2}-r^{2}\right]^{11^{2}}(n-r)
$$


For closure domains to form $w_{\text {ans }} \leqslant w_{\text {mag }}$ here $w_{\text {mas }}$ is the second term in (5). The condition for the formation of closure domains is then given by

$$
M_{2}^{2}=\frac{9}{\pi} \frac{k}{n} \frac{K}{(n+1)^{2}}\left(M_{\mathrm{s}} / M_{0}\right) \sum_{r=1}^{n}\left[(n+1)^{2}-r^{2}\right]^{1 / 2}(n-r) .
$$

For a given value of $M_{v}, R$ and $\sigma_{w}, n$ is obtained using (6). This value is substituted in (10) and the value of $k$ is estimated after numerical summation. This yields a reasonable value of 0.25 for $k$.

The mixed spectrum is a manifestation of simultaneous presence of grains of radius for which closure domain may or may not be permitted. We have thus obtained a method by which we can explain the existence of the different types of permeability spectra and the parameters which control the narure of these spectra.

\section{References}

Brown F and Gravel C L 1955 Phys. Rev. 9755

Chikazumi S and Charap S H 1964 Physics of Magnetism (New York : John Wiley) p. 186

Globus A 1962 C.R. Acad. Sci. (Paris) 2551709

Globus A 1975 European Physical Society Conference on Soft Magnetic Materials (Cardiff) p. 2

Globus A 1976 Int. Conf. Ferrites 2 Bellevue, France, Suppl. to J. Phys. C 1-1 (1976)

Kittel C and Galt J K 1956 Solid State Physics (eds F Seitz and D Turnbull) (New York: Academic Press) 3437

Malinofsky W W and Babbit R W $1961 \mathrm{~J}$. Appl. Phys. 322375

Rado G T 1953 Rev. Mod. Phys. 2581

Rado G T, Wright R W and Emerson W H 1950 Phys. Rev. 80273

Smit J and Wijn H P J 1959 Ferrites (New York : John Wiley) p. 27

Smolenski G A, Tszun C and Stankevich A K 1961 Sov. Phys. (Solid State) 3486

Srivastava C M, Shringi S N, Srivastava R G and Nanadikar 1976 Phys. Rey. B 142032

Verweel J 1962 Proc. Inst. Electr. Engr. B 109 Suppl. to 2195 